\title{
THE CORRELATION COEFFICIENTS OF THE QUEUE LENGTHS OF SOME STATIONARY SINGLE SERVER QUEUES
}

\author{
A. G. PAKES \\ (Received 30 September 1968; revised 19 June 1969) \\ Communicated by P. D. Finch
}

\section{Introduction}

Until recently there has been little systematic work on the second-order properties of queueing processes. The aim of this paper is to study systematically the second-order properties of the queue length processes embedded at departure epochs in the $M / G / 1$ and bulk service $M / G / 1$ queues, and at arrival epochs in the $G I / M / 1$ queue. In the latter case our results extend those of Daley [7], while in the ordinary $M / G / 1$ queue our work parallels Daley's [6] discussion of waiting times in the same system. In the final section we briefly discuss two discrete time queueing systems.

Much of the earlier work on second-order properties of queueing systems has been devoted to continuous time processes. Morse [12] obtained the autocorrelation function for the continuous time queue length process in the stationary $M / M / 1$ queue. Observation of this function shows it to be completely monotone. Parzen [14] (equations 5.25 and 5.26, p. 149) has given this function for the stationary $M / G / \infty$ queue and it can be seen to be a convex function, and completely monotonic for $M / M / \infty$. Beneš [1] obtained the autocovariance function for the virtual waiting time in the stationary $M / G / 1$ queue. More recently, Reynolds [16] has given a more general approach to the calculation of the autocovariance function of the continuous time queue length process in some stationary queueing models, including an approach to some systems with finite waiting rooms. $\mathrm{He}$ illustrates his results by considering the stationary $M / M / 1$ queue, the $M / M / 1$ queue with finite waiting room and a batch arrival infinite server queue.

Some of the more recent work concerns discrete time processes. The first work in this direction seems to be that of Craven [5] who calculated the serial correlation coefficients of lags one, two, and three for a stationary sequence of waiting times in the $M / M / 1$ queue. On the basis of the heavy traffic form of his results, he made a conjecture as to the heavy traffic form of the entire sequence of serial correlation coefficients. Blomqvist [3] has considered the serial correlation coefficients of a stationary sequence of waiting times in $M / G / 1$ from the point of view of simulating 
such systems. Daley [6] has given a systematic treatment of the serial correlation coefficients of a stationary sequence of waiting times in the $G I / G / 1$ queue and in particular the $M / G / 1$ queue, for which he proves a generalization of Craven's conjecture. Jenkins [9] has calculated the correlation coefficient of lag one for a stationary sequence of the embedded queue length process in the $M / E_{k} / 1$ queue. Daley [7] has dealt with the queue length problem of the stationary $G I / M / 1$ queue from the point of view of simulation.

At this point, it is worth noting that some of the notation will be used to denote different quantities throughout the paper. However, within any section there will be no ambiguity.

\section{The stationary $M / G / 1$ queue}

In a stationary $M / G / 1$ queueing system with Poisson arrivals at rate $\lambda$, service distribution function $B(\cdot)$, traffic intensity. $\rho=\lambda \mu_{1}<1$ where $\mu_{r}=\int_{0}^{\infty} x^{r} d B(x)$ $(r=1,2, \cdots)$, we consider the embedded process $\left\{Q_{n}\right\}(n=\cdots,-1,0,1, \cdots)$ of the length of the queue at successive departure epochs of customers. As is well known (Takács [18]), $\left\{Q_{n}\right\}$ is an ergodic Markov chain with one-step transition probabilities $p_{i j}=k_{j+1-i}(1 \leqq i \leqq j+1), p_{0 j}=p_{1 j}$, and $p_{i j}=0$ otherwise $(i, j=0,1, \cdots)$, where $K(x)=\sum_{j=0}^{\infty} k_{j} x^{j}=\int_{0}^{\infty} e^{-\lambda t(1-x)} d B(t)=\beta[\lambda(1-x)]$ $(|x| \leqq 1)$ so that $\rho=K^{\prime}(1)$. The stationary distribution $\left\{\pi_{i}\right\}$ for the Markov chain $\left\{Q_{n}\right\}$ has the generating function

$$
\Pi(x)=\sum_{i=0}^{\infty} \pi_{i} x^{i}=\frac{(1-\rho)(1-x) K(x)}{K(x)-x} \quad(|x| \leqq 1)
$$

from which the moments $v_{r}=E\left(Q_{r}^{n}\right)($ all $n, r=1,2, \cdots)$ are easily found;

$$
\begin{aligned}
v_{1} & =\rho+\lambda \mu_{2} / 2(1-\rho) \\
v_{2} & =\rho+\frac{\lambda^{3} \mu_{3}}{3(1-\rho)}+\frac{\lambda^{4} \mu_{2}^{2}}{2(1-\rho)^{2}}+\frac{3 \lambda^{2} \mu_{2}}{2(1-\rho)} \\
\sigma^{2} & =-\rho(1-\rho)+\frac{\lambda^{3} \mu_{3}}{3(1-\rho)}+\frac{1}{4}\left[\frac{\lambda^{2} \mu_{2}}{1-\rho}\right]^{2}+\frac{3-2 \rho}{2(1-\rho)} \lambda^{2} \mu_{2}
\end{aligned}
$$

where $\sigma^{2}=\operatorname{var}\left(Q_{n}\right)($ all $n)$. It is easily seen that $v_{r}$ is finite iff $\mu_{r+1}$ is; we shall always assume $\mu_{3}<\infty$.

Define the serial correlation coefficients $r_{n}=\left(M_{n}-v_{1}^{2}\right) / \sigma^{2}$ where $M_{n}=$ $E\left(Q_{m} Q_{m+n}\right)$ (all $\left.m ; n=0,1, \cdots\right)$. By the Cauchy-Schwartz inequality $\left\{r_{n}\right\}$ is well defined when $\mu_{3}<\infty$. Appealing to the following theorem, we see that $\left\{r_{n}\right\}$ is a monotone non-increasing sequence with limit zero. This theorem is a special case of a theorem due to Daley [8]; see also the remarks on p. 313 of [8] and Pakes [13].

THeOREM D. Let $\left\{X_{n}\right\}(n=\cdots,-1,0,1, \cdots)$ be a stationary ergodic Markov chain on the non-negative integers and let it be stochastically monotone, that is 
$\operatorname{Pr}\left\{X_{n+1} \leqq j \mid X_{n}=i\right\} \geqq \operatorname{Pr}\left\{X_{n+1} \leqq j \mid X_{n}=i+1\right\}$ (all $\left.n ; i, j=0,1, \cdots\right)$ and let $E\left\{X_{0}^{2}\right\}<\infty$. Then the serial correlation coefficients $r_{n}=\left[E\left(X_{m} X_{m+n}\right)-\left(E\left(X_{m}\right)\right)^{2}\right] /$ $\operatorname{var}\left(X_{m}\right)($ all $m ; n=0,1, \cdots)$ form a monotone non-increasing sequence with limit equal to zero.

We now prove Theorem 1 which is the key to further properties of the serial correlation coefficients $\left\{\boldsymbol{r}_{\boldsymbol{n}}\right\}$.

THEOREM 1. The generating function $R(y)=\sum_{n=0}^{\infty} r_{n} y^{n}(|y|<1)$ of the serial correlation coefficients $\left\{r_{n}\right\}$ of the embedded queue length process $\left\{Q_{n}\right\}$ of the stationary $M / G / 1$ queue with $\mu_{3}<\infty$, is given by

$$
R(y)=\frac{1}{1-y}-\frac{v_{1}(1-\rho) y}{\sigma^{2}(1-y)^{2}}+\frac{y H(0, y)}{\sigma^{2}(1-y)}
$$

where $H(0, y)=\sum_{n=1}^{\infty} C_{n} y^{n}(|y|<1)$ and where with $D \equiv d / d x$,

(4) $C_{n}=E\left(Q_{0} ; Q_{n}=0\right)=\left.\frac{1}{n !} D^{n-1}\left\{D\left[\frac{x \Pi^{\prime}(x)}{1-x}\right][K(x)]^{n}\right\}\right|_{x=0}(n=1,2, \cdots)$.

The correlation coefficients are given by

$$
\begin{aligned}
& r_{1}=1-v_{1}(1-\rho) / \sigma^{2}, \\
& r_{n}=1-n v_{1}(1-\rho) / \sigma^{2}+\left(\sum_{k=1}^{n-1} C_{k}\right) / \sigma^{2} \quad(n=2,3, \cdots) .
\end{aligned}
$$

The sequence $\left\{r_{n}\right\}$ is convex.

Proof. Defining $\theta_{j}^{(n)}=\sum_{i=1}^{\infty} i \operatorname{Pr}\left\{Q_{0}=i, Q_{n}=j\right\}=E\left\{Q_{0} ; Q_{n}=j\right\}(n, j=$ $0,1, \cdots)$, we see that $\theta_{j}^{(n+1)}=\sum_{i=0}^{\infty} \theta_{i}^{(n)} p_{i j}$ which for $M / G / 1$ is

$$
\theta_{j}^{(n+1)}=k_{j} \theta_{0}^{(n)}+\sum_{i=0}^{j} k_{j-i} \theta_{i+1}^{(n)}
$$

Forming the generating functions $H_{n}(x)=\sum_{j=0}^{\infty} \theta_{j}^{(n)} x^{j}=E\left(Q_{0} x^{Q_{n}}\right)(|x| \leqq 1)$ gives us

$$
x H_{n+1}(x)-K(x) H_{n}(x)=-\theta_{0}^{(n)} K(x)(1-x) \quad(n=0,1, \cdots ;|x| \leqq 1)
$$

Taking generating functions again, and observing that

gives

$$
H_{0}(x)=\sum_{j=1}^{\infty} j \pi_{j} x^{j}=x \Pi^{\prime}(x)
$$

(6) $H(x, y)=\sum_{n=0}^{\infty} H_{n}(x) y^{n}=\sum_{n=0}^{\infty} E\left(Q_{0} x^{Q_{n}}\right) y^{n}=\frac{x^{2} \Pi^{\prime}(x)-(1-x) y K(x) H(0, y)}{x-y K(x)}$

$$
(|x| \leqq 1,|y|<1)
$$

where $H(0, y)=\sum_{n=0}^{\infty} C_{n} y^{n}$ on putting $\theta_{0}^{(n)} \equiv C_{n}(n=0,1, \cdots)$. 
Applying Abel's theorem, as given by Karlin [10] p. 463 to (6) shows that $M(y)=\sum_{n=0}^{\infty} M_{n} y^{n}=\partial H(x, y) /\left.\partial x\right|_{x=1}$, where the derivative at $x=1$ is taken through positive $x<1$. (This convention will be adhered to throughout the paper.) Simple algebra then leads to (3). Using the uniqueness theorem for power series and expanding the right hand side of (3) gives (5). We now demonstrate (4) by means of Lagrange's theorem for the reversion of series.

First note that the function $x-y K(x)$ has, for each $0 \leqq y<1$, a unique zero $\tau(y)$ in the unit circle; see Takacs [18] p. 47 and p. 235. But from its definition $H(x, y)$ is a regular function of $x$ in the open unit disc for each $0 \leqq y<1$, so that the numerator of (6) has a zero at $x=\tau(y)$ and this implies that

$$
H(0, y)=\frac{\tau(y) \Pi^{\prime}[\tau(y)]}{1-\tau(y)}
$$

By observing that $x \Pi^{\prime}(x) /(1-x)$ is regular in the open unit disc, Lagrange's theorem gives (4).

The convexity of $\left\{r_{n}\right\}$ follows on noting that (5) implies $\sigma^{2}\left(r_{n}-2 r_{n+1}+r_{n+2}\right)$ $=C_{n+1}-C_{n}$, and since $\left\{Q_{n}\right\}$ is a stochastically monotone Markov chain, $\left\{C_{n}\right\}_{0}^{\infty}$ is a monotone non-decreasing sequence; see Theorem 3 of Pakes [13]. The proof is now complete.

In order to estimate the mean of a stationary stochastic process such as is the queue length process $\left\{Q_{n}\right\}$, given a sample of observations $\left(Q_{1}, \cdots, Q_{N}\right)$, we form the unbiassed estimator $m=\left(\sum_{n=1}^{N} Q_{n}\right) / N$ whose variance is given exactly by

$$
\operatorname{var}(m)=\sigma^{2}\left\{N+2 \sum_{n=1}^{N-1}(N-n) r_{n}\right\} / N^{2} .
$$

If $\sum_{n=0}^{\infty} r_{n}$ converges, the variance of the sample mean is, when $N$ is large, asymptotically equal to

$$
\sigma^{2}\left\{1+2 \sum_{n=1}^{\infty} r_{n}\right\} / N
$$

Thus for the purposes of estimation of $v_{1}$, we are interested in the convergence of $\sum_{n=0}^{\infty} r_{n}$, and it follows from the non-negativity of $r_{n}$ and Abel's theorem that $\sum_{n=0}^{\infty} r_{n}<\infty$ iff $\lim _{y \uparrow 1} R(y)<\infty$, and the two limits are equal. The following theorem deals with this matter.

THEOREM 2. $\sum_{n=0}^{\infty} r_{n}$ converges iff $\mu_{4}<\infty$ and when this condition is satisfied,

$$
\sum_{n=0}^{\infty} r_{n}=1-\frac{1}{2(1-\rho)}+\frac{v_{3}-v_{2} v_{1}}{2(1-\rho) \sigma^{2}}
$$

Proof. Write $R(y)$ in the form

$$
R(y)=\left[\sigma^{2}(1-y)-v_{1}(1-\rho) y+y G(y)\right] / \sigma^{2}(1-y)^{2}
$$

where 


$$
G(y)=(1-y) H(0, y)=(1-\rho) y \tau(y) \Pi^{\prime}[\tau(y)](\Pi[\tau(y)]-(1-\rho)(1-\tau(y)))^{-1}
$$

and where the second equality comes from (1) and (7). This form of $G(y)$ is convenient to handle. A two fold application of L'Hospitals rule to (10) shows that

$$
\sum_{n=0}^{\infty} r_{n}=R(1)=\lim _{y \uparrow 1}\left[2 G^{\prime}(y)+G^{\prime \prime}(y)\right] / 2 \sigma^{2}
$$

It can be seen from (11) that $G^{\prime \prime}(y)$ will involve $d^{3} \Pi(x) /\left.d x^{3}\right|_{x=\tau(y)}$ and no higher derivatives, and since $\tau(1)=1$, it follows by monotone convergence that this third order derivative tends, as $y \uparrow 1$, to $E\left\{Q_{n}\left(Q_{n}-1\right)\left(Q_{n}-2\right)\right\}$. Thus $R(1)$ is finite iff $v_{3}<\infty$ and this latter condition holds iff $\mu_{4}<\infty$. Equation (9) follows on noting that

$$
G^{\prime}(1)=\sigma^{2} \quad \text { and } \quad G^{\prime \prime}(1)=\left(v_{3}-v_{2} v_{1}-\sigma^{2}\right) /(1-\rho) .
$$

This theorem should be contrasted with Theorems 2 and 4 of Daley [6] where he shows that the sum of the serial correlation coefficients of a stationary sequence of waiting times in $G I / G / 1$ is finite iff the fourth moment of the service distribution is finite.

COROLLARY 1. Let $\left\{Q_{n}\right\}_{n=1}^{N}$ be an observed sequence of queue lengths in the stationary $M / G / 1$ queue with $\mu_{4}<\infty$. Then for $N$ large

$$
\operatorname{var}(m) \approx\left(v_{3}-v_{2} v_{1}-\rho \sigma^{2}\right) / N(1-\rho)
$$

Proof. This follows from (8) and Theorem 2.

COROLlary 2. Under the conditions of Theorem 2,

$$
1+2 \sum_{n=1}^{\infty} r_{n} \sim \frac{2 \lambda^{2} \mu_{2}}{(1-\rho)^{2}}+\frac{2 \lambda \mu_{3}}{3 \mu_{2}(1-\rho)}+\frac{1+\rho}{1-\rho}+O(1) \quad(\rho \uparrow 1)
$$

Proof. This follows from Theorem 2, equations (2) and by using

$$
v_{3}=\frac{\lambda^{4} \mu_{4}}{4(1-\rho)}+\frac{\lambda^{5} \mu_{2} \mu_{3}}{(1-\rho)^{2}}+\frac{3 \lambda^{6} \mu_{2}^{3}}{4(1-\rho)^{3}}+\frac{3 \lambda^{4} \mu_{2}^{2}}{(1-\rho)^{2}}+\frac{7 \lambda^{2} \mu}{2(1-\rho)}+\frac{3 \lambda^{3} \mu_{3}}{1-\rho}+\rho
$$

which is easily found from (1).

Comparing Corollary 2 above with Corollary 4.2 of Daley [6], we see that when $\mu_{4}<\infty$ in the stationary $M / G / 1$ queue $1+2 \sum_{n=1}^{\infty} r_{n}$ is asymptotically equal $(\rho \uparrow 1)$ to the corresponding expression for a stationary sequence of waiting times. We now prove a theorem which shows that the heavy traffic behaviour of $\left\{\boldsymbol{r}_{\boldsymbol{n}}\right\}$ is similar to that of the waiting time serial correlation coefficients; see Daley [6], Theorem 5.

THEOREM 3. For the family of ergodic $M / G / 1$ queueing systems with given service time distribution $B(\cdot)$ and arrival rate $\lambda$ such that $0<1-\rho<\delta<1$ and 
$\mu_{3}<\infty$, the serial correlation coefficients $r_{n}$ of a stationary sequence of queue lengths $\left\{Q_{n}\right\}$ satisfy

$$
\left|r_{n}-1+A n(1-\rho)^{2}\right|<M(1-\rho)^{3}
$$

for any finite set of integers $n=1, \cdots, N$, where $A=2 / \lambda^{2} \mu_{2}$ and $M$ is a constant depending on $N$.

Proof. From (2) it is easy to show that $v_{1} / \sigma^{2}=2(1-\rho) / \lambda^{2} \mu_{2}+O\left[(1-\rho)^{2}\right]$ ( $\rho \uparrow 1$ ) so that the theorem is true for $N=1$. In fact, since $1 / \sigma^{2}=O\left[(1-\rho)^{2}\right]$ $(\rho \uparrow 1)$, it is clear from (5) that we only need to show that there is a constant $M$ such that $\sum_{k=1}^{N-1} C_{k}<M(1-\rho)$.

The generating function $K(x)$ is given by $\beta[\lambda(1-x)]$, where $\beta(s)$, the LaplaceStieltjes transform of the service time distribution function $B(\cdot)$, is a function of $s$ regular in $\operatorname{Re} s>0$. So for each positive $\lambda, \beta[\lambda(1-x)]$ is a function of $x$ regular in $S_{x}=\{x:|x|<\varepsilon<1\}$ and for each such $x, \beta[\lambda(1-x)]$ is a function of $\lambda$ uniformly continuous in the set $S_{\lambda}=\left\{\lambda:(1-\delta) / \mu_{1} \leqq \lambda \leqq\left(1+\varepsilon_{1}\right) / \mu_{1}\right\}\left(\varepsilon_{1}>0\right)$.

Using (1) it is easily seen that

where

$$
x \Pi^{\prime}(x) /(1-x)=(1-\rho) W(x, \lambda) \quad(0<\rho<1,0 \leqq x \leqq 1)
$$

$$
W(x, \lambda)=\frac{\lambda x^{2}(1-x) \beta^{\prime}[\lambda(1-x)]-x \beta^{2}[\lambda(1-x)]+x \beta[\lambda(1-x)]}{(1-x)[x-\beta(\lambda(1-x))]^{2}} .
$$

Setting $\varepsilon<\beta\left[2\left(1+\varepsilon_{1}\right) / \mu_{1}\right]$, it is clear that $x-\beta[\lambda(1-x)]$ has no zeros in $S=$ $S_{x} \times S_{\lambda}$, and so under this condition it follows from the regularity of $\beta(\cdot)$ that $W(x, \lambda)$ is a function of $x$ regular in $S_{x}$ for each $\lambda \in S_{\lambda}$, and it is a function of $\lambda$ uniformly continuous in $S_{\lambda}$ for each $x \in S_{x}$.

Now $(1-\rho)^{-1} \sum_{k=1}^{n-1} C_{k}$ is a finite sum of finite order derivatives of a finite product of functions regular in $S_{x}$ for each fixed $\lambda \in S_{\lambda}$ and uniformly continuous in $S_{\lambda}$ for each $x \in S_{x}$. Hence a finite set of such sums is uniformly bounded in each compact subset of $S_{x} \times S_{\lambda}$. Taking $\left\{(s, \lambda): s=0, \lambda \in S_{\lambda}\right\}$ as such a set, we see that for $n=1, \cdots, N$ there exists a constant $M$ such that $\sum_{k=1}^{n-1} C_{k}<M(1-\rho)$, so the proof is complete.

\section{The stationary $M / M / 1$ queue}

We shall illustrate the results of the previous section by discussing the stationary $M / M / 1$ queue, that is $B(x)=1-e^{-x / \mu 1}$, for which the algebra is tractable. The results of this algebra are

$$
\begin{aligned}
K(y) & =(1+\rho-\rho y)^{-1}, \quad \Pi(y)=(1-\rho) /(1-\rho y), \\
\tau(y) & =\left(1+\rho-\left[(1+\rho)^{2}-4 \rho y\right]^{\frac{1}{2}}\right) / 2 \rho, \\
H(0, y) & =\frac{1-\rho^{2}}{2 \rho(1-y)^{2}}\left[\sqrt{1-a y}-1+\frac{2 \rho y}{1+\rho}\right] \quad \text { where } a=\frac{4 \rho}{(1+\rho)^{2}},
\end{aligned}
$$




$$
\begin{aligned}
C_{n} & =\frac{1-\rho^{2}}{2 \rho}\left(\frac{2 \rho n}{1+\rho}+\sum_{r=1}^{n}(n+1-r)\left(\begin{array}{l}
\frac{1}{2} \\
r
\end{array}\right)(-a)^{r}\right) \\
R(y) & =\frac{1}{1-y}-\frac{y(1-\rho)^{2}}{(1-y)^{2}}+\frac{y(1+\rho)(1-\rho)^{3}}{2 \rho^{2}(1-y)^{3}}\left[\sqrt{1-a y}-1+\frac{2 \rho y}{1+\rho}\right]
\end{aligned}
$$

By working as in Daley [6] p. 697, we can obtain the representation

$$
r_{\mathrm{n}}=\frac{(1-\rho)^{3}(1+\rho)}{2 \pi \rho^{2}} \int_{0}^{a} t^{n+\frac{1}{2}} \frac{(a+t)^{\frac{1}{2}}}{(1-t)^{3}} d t=\frac{(1-\rho)^{3}(1+\rho) a^{n+2}}{2 \pi \rho^{2}} \int_{0}^{1} u^{n+\frac{1}{2}} \frac{(1-u)^{\frac{1}{2}}}{(1-a u)^{3}} d u
$$

for $n=0,1, \cdots$. This shows that the sequence $\left\{r_{n}\right\}$ is completely monotone. It should be noted that equation (34) in [6] is in error; the left hand side should read $\rho_{n-1}$. The error is made in the first equation of p. 697; compare with equation (21) of [6].

Again, working as in Daley [6] we have

$$
r_{n}=O\left(a^{n} n^{n-\frac{1}{2}}\right) \quad(n \rightarrow \infty)
$$

From Theorem 3, we have the heavy traffic approximation $r_{n}=1-n(1-\rho)^{2}$ $+O\left[(1-\rho)^{3}\right](\rho \uparrow 1)$. We can find a light traffic approximation in the following manner. Observing that the terms of the series $(1-a u)^{-3}=\sum_{r=0}^{\infty}\left(\begin{array}{c}2+r \\ r\end{array}\right)(a u)^{r}$ are non-negative enables us to integrate term by term and so (12) yields.

$$
r_{n}=\frac{(1-\rho)^{3}(1+\rho) a^{n+2}}{2 \pi \rho^{2}} \sum_{r=0}^{\infty}\left(\begin{array}{c}
2+r \\
r
\end{array}\right) a^{r} B\left(n+r+\frac{3}{2}, \frac{3}{2}\right)
$$

Taking the first term of this series, and retaining only the lowest power of $\rho$ shows that

$$
r_{n}=A_{n} \rho^{n}+O\left(\rho^{n+1}\right) \quad(\rho \downarrow 0)
$$

where $A_{n}=2^{n+1} \cdot 1 \cdot 3 \cdots(2 n+1) /(n+2)$ !

\section{4, The bulk service queue}

In a stationary bulk service queue with Poisson arrivals at rate $\lambda$, the customers are served in batches of size $s$ or less, where the positive integer $s$ is fixed, with the batch service time distribution function $B(\cdot)$ and traffic intensity $\rho=\lambda \mu_{1} / s<1$ where $\mu_{r}=\int_{0}^{\infty} x^{r} d B(x)$. We consider the embedded process $\left\{Q_{n}\right\}(n=\cdots,-1,0$, $1, \cdots)$ of the length of the queue at successive departure epochs of the batches. As is well known (Saaty [17]) $\left\{Q_{n}\right\}$ is an ergodic Markov chain with one step transition probabilities $p_{i j}=k_{j-i+s}(s \leqq i \leqq j+s), p_{i j}=p_{s j}(0 \leqq i \leqq s)$, and $p_{i j}=0$ otherwise $(i, j=0,1, \cdots)$, where $K(x)=\sum_{j=0}^{\infty} k_{j} x^{j}=\int_{0}^{\infty} e^{-\lambda t(1-x)} d B(t)$ $(|x| \leqq 1)$, so that $\rho=K^{\prime}(1) / s$. The stationary distribution $\left\{\pi_{i}\right\}$ for the Markov chain $\left\{Q_{n}\right\}$ has generating function 


$$
\begin{aligned}
\Pi(x) & =\sum_{i=0}^{\infty} \pi_{i} x^{i}=K(x)\left\{\sum_{i=0}^{s-1} \pi_{i}\left(x^{s}-x^{i}\right)\right\} /\left(x^{s}-K(x)\right) \\
& =\frac{s(1-\rho)(1-x) K(x)}{K(x)-x^{s}} \prod_{i=1}^{s-1} \frac{x-\tau_{i}}{1-\tau_{i}} \quad(|x| \leqq 1)
\end{aligned}
$$

where $\left\{\tau_{i}\right\}_{1}^{s-1}$ are the $s-1$ solutions within the unit circle of the equation $x^{s}-K(x)$ $=0$.

Letting $v_{r}=E\left(Q_{n}^{r}\right) \quad($ all $n ; r=1,2, \cdots)$ and $\sigma^{2}=v_{2}-v_{1}^{2}$ and noting that $v_{r}$ is finite iff $\mu_{r+1}$ is, we see that the serial correlation coefficients $r_{n}=\left[E\left(Q_{0} Q_{n}\right)-v_{1}^{2}\right] / \sigma^{2}$ are well defined if $\mu_{3}<\infty$. Observing that the Markov chain $\left\{Q_{n}\right\}$ is stochastically monotone, Theorem $\mathbf{D}$ shows that the serial correlation coefficients of the embedded queue length process $\left\{Q_{n}\right\}$ of the stationary bulk service queue, are monotone non-increasing with limit equal to zero. The remainder of this section is devoted to finding a representation for $\left\{\boldsymbol{r}_{\boldsymbol{n}}\right\}$ and showing that it is a convex sequence.

THEOREM 4. The generating function $R(-y)=\sum_{n=0}^{\infty} r_{n} y^{n}(|y|<1)$ of the serial correlation coefficients $\left\{r_{n}\right\}$ of the embedded queue length process $\left\{Q_{n}\right\}$ of the stationary bulk service queue with $\mu_{3}<\infty$ is given by

$$
R(y)=\frac{1}{1-y}-\frac{s v_{1}(1-\rho) y}{\sigma^{2}(1-y)^{2}}+\frac{y P(1, y)}{\sigma^{2}(1-y)}
$$

where

and

$$
P(1, y)=\sum_{n=0}^{\infty} O_{n} y^{n}=\sum_{i=1}^{s} g_{i}(y) l_{i}(1, y) \quad(|y|<1)
$$

$$
\begin{aligned}
g_{i}(y) & =\tau_{i}(y) \Pi^{\prime}\left[\tau_{i}(y)\right] /\left(1-\tau_{i}(y)\right), \\
l_{i}(1, y) & =\prod_{j=1}^{s-1}\left(1-\tau_{j}(y)\right] /\left[\tau_{i}(y)-\tau_{j}(y)\right] .
\end{aligned}
$$

The $\tau_{i}(y)(i=1, \cdots, s)$ are the soots within the unit circle of the equation

$$
x^{s}-y K(x)=0 \quad(|y|<1) .
$$

The correlation coefficients are given by

$$
r_{n}=1-s v_{1} n(1-\rho) / \sigma^{2}+\left(\sum_{m=0}^{n-1} O_{m}\right) / \sigma^{2} \quad(n=1,2, \cdots) .
$$

The sequence $\left\{r_{n}\right\}$ is convex.

Proof. Letting $\theta_{j}^{(n)}=E\left(Q_{0} ; Q_{n}=j\right)$ we see that

$$
\theta_{j}^{(n+1)}=\sum_{i=0}^{\infty} \theta_{i}^{(n)} p_{i j} \quad(n, j=0,1, \cdots)
$$

and proceeding as in Theorem 1 we find, if $|x| \leqq 1$ and $|y|<1$, 


$$
H(x, y)=\sum_{n=0}^{\infty} E\left(Q_{0} x^{Q_{n}}\right) y^{n}=\frac{x^{s+1} \Pi^{\prime}(x)+y K(x) \sum_{j=0}^{s-1} h_{j}(y)\left(x^{s}-x^{j}\right)}{x^{s}-y K(x)},
$$

where $h_{j}(y)=\sum_{n=0}^{\infty} \theta_{j}^{(n)} y^{n}$. It follows by inspection that

$$
\sum_{j=0}^{s-1} h_{j}(y)\left(x^{s}-x^{j}\right)=-(1-x) P(x, y)
$$

where $P(x, y)$ is a polynomial in $x$ of degree $s-1$. The assertion concerning the roots of (15) are well known; see Takács [18] p. 82, and for $i=1, \cdots, s-1$, $\tau_{i}(1)=\tau_{i}$ and $\tau_{s}(1)=1$ when the $\tau_{i}$ and $\tau_{i}(y)$ are suitably ordered.

For each $y$ in $|y|<1, H(x, y)$ is a function of $x$ regular in the unit disc, so that the numerator of $(17)$ has $s$ zeros coinciding with those of the denominator, so Lagrange's interpolation formula (Cheney [4] p. 58) shows that

where $g_{i}(y)$ is given by (14) and

$$
P(x, y)=\sum_{i=1}^{s} g_{i}(y) l_{i}(x, y)
$$

$$
l_{i}(x, y)=\prod_{j=1}^{s-1}\left(x-\tau_{j}(y)\right) /\left(\tau_{i}(y)-\tau_{j}(y)\right) .
$$

Observing that $x^{s}-x^{j}=-(1-x)\left(x^{s-1}+x^{s-2}+\cdots+x^{j}\right)(j=0,1, \cdots, s-1)$ and using the defining relations for $P(x, y)$ shows that

$$
P(x, y)=\sum_{j=0}^{s-1} L_{j}(y) x^{j}
$$

where $L_{j}(y)=\sum_{n=0}^{\infty} \phi_{j}^{(n)} y^{n}$ and where $\phi_{j}^{(n)}=E\left(Q_{0} ; Q_{n} \leqq j\right)$. Abel's theorem enables (13) to be found from (17) and (18), and (16) follows from (13) and the uniqueness of power series expansions. Observing that $\sigma^{2}\left(r_{n+2}-2 r_{n+1}+r_{n}\right)=$ $O_{n+1}-O_{n}$ and that $O_{n}=\sum_{j=0}^{s-1} \phi_{j}^{(n)}$ (from (19)), it follows from the stochastic monotonicity of the Markov chain $\left\{Q_{n}\right\}$ that $\left\{r_{n}\right\}$ is a convex sequence; see Theorem 3 of Pakes [13].

\section{The stationary $G I / M / 1$ queue}

In a stationary $G I / M / 1$ queueing system with service times distributed exponentially with parameter $\mu$ and with independent interarrival times having distribution function $A(\cdot)$ and traffic intensity $\rho=\left(\mu \lambda_{1}\right)^{-1}<1$, where $\lambda_{r}=$ $\int_{0}^{\infty} x^{r} d A(x)(r=1,2, \cdots)$, we consider the embedded process $\left\{Q_{n}\right\}(n=\cdots,-1$, $0,1, \cdots)$ of the queue length at successive arrival epochs of customers. As is well known (Takács [18]) $\left\{Q_{n}\right\}$ is an ergodic Markov chain with one-step transition probabilities $p_{i j}=f_{i+1-j}(1 \leqq j \leqq i+1) p_{i 0}=1-\sum_{k=0}^{i} f_{k}$ and $p_{i j}=0$ otherwise $(i, j=0,1, \cdots)$, where 


$$
F(x)=\sum_{i=0}^{\infty} f_{i} x^{i}=\int_{0}^{\infty} e^{-\mu(1-x) t} d A(t) \quad(|x| \leqq 1), \text { so that } \rho^{-1}=F^{\prime}(1) .
$$

The stationary distribution $\left\{\pi_{i}\right\}$ for the Markov chain $\left\{Q_{n}\right\}$ is given by $\pi_{i}=$ $(1-\xi) \xi^{i}(i=0,1, \cdots)$ where $\xi$ is the unique root in $(0,1)$ of the equation $F(x)=x$.

Letting $v_{r}=E\left(Q_{n}^{r}\right)$ (all $n ; r=1,2, \cdots$ ) and $\sigma^{2}=v_{2}-v_{1}^{2}$, we define the serial correlation coefficients $r_{n}=\left(E\left(Q_{0} Q_{n}\right)-v_{1}^{2}\right) / \sigma^{2}$. As Daley [7] has pointed out, $\left\{Q_{n}\right\}$ is a stochastically monotone Markov chain and $\left\{r_{n}\right\}$ is a monotone nonincreasing sequence with limit equal to zero. Daley has also found an expression for the generating function $R(y)=\sum_{n=0}^{\infty} r_{n} y^{n}(|y|<1)$;

$$
R(y)=\frac{1}{1-y}-\frac{y \xi(1-\xi)}{(1-y)^{2} \xi^{\prime}(1)}+\frac{y \xi(y)(1-\xi)^{2}}{\xi^{\prime}(1)(1-y)^{2}(1-\xi(y))^{2}}
$$

where $\xi(y)$ is the root of smallest absolute value of the equation $x=y F(x)$, $\xi=\xi(1)$ and $\xi^{\prime}(1)=d /\left.d y \xi(y)\right|_{y=1}$. He has shown that $R(1)$ is finite and he found a heavy traffic approximation for $R(1)$. The following theorem exhibits the form of $\left\{r_{n}\right\}$ and gives further monotone properties.

THEOREM 5. The serial correlation coefficients $\left\{\boldsymbol{r}_{n}\right\}$ of the embedded queue length process of the stationary $G I / M / 1$ queue are given by

$$
r_{n}=1-n(1-\xi) \xi / \xi^{\prime}(1)+(1-\xi)^{2}\left(\sum_{j=0}^{n-1}(n-j) \beta_{j}\right) / \xi^{\prime}(1) \quad(n=1,2, \cdots)
$$

where $\beta_{0}=0$ and where, with $D \equiv d / d x$,

$$
j ! \beta_{j}=\left.D^{j-1}\left[F^{j}(x) /(1-x)^{2}\right]\right|_{x=0} \quad(j=1,2, \cdots) .
$$

The sequence $\left\{r_{n}\right\}$ is convex and its third differences are non-positive i.e.

$$
r_{n+3}-3 r_{n+2}+3 r_{n+1}-r_{n} \leqq 0 \quad(n=0,1, \cdots) .
$$

Proof. Lagrange's theorem shows that $\xi(y) /(1-\xi(y))$ is power series expansible within the unit disc, so letting $\xi(y) /(1-\xi(y))=\sum_{j=0}^{\infty} \beta_{j} y^{j}(|y|<1)$, (21) and (22) follow immediately. We see from (21) that $r_{n+2}-2 r_{n+1}+r_{n}=\beta_{n+1}$ $(n=0,1, \cdots)$, so that the convexity of $\left\{r_{n}\right\}$ is established on observing that both $F(x)$ and $(1-x)^{-2}$ have power series expansions with positive coefficients, so $\beta_{n} \geqq 0(n=1,2, \cdots)$.

The final statement of the theorem will be proved if we can show that $\left\{\beta_{n}\right\}_{1}^{\infty}$ is a monotone non-increasing sequence. Let

$$
f_{00}^{(n)}=\operatorname{Pr}\left\{Q_{n}=0, Q_{m} \neq 0(m=1, \cdots, n-1) \mid Q_{0}=0\right\} \quad(n=1,2, \cdots) .
$$

Then we have $\sum_{n=1}^{\infty} f_{00}^{(n)} y^{n}=(y-\xi(y)) /(1-\xi(y))(|y|<1)$; see Takács [18] p. 116 . From this we find that $f_{00}^{(1)}=1-\beta_{1} \geqq 0$ and $f_{00}^{(n)}=\beta_{n-1}-\beta_{n} \geqq 0(\mathrm{n}=2,3, \cdots)$, thus completing the theorem. 
Let

$$
F^{n}(x)=\sum_{j=0}^{\infty} f_{j}^{(n)} x^{j} \quad(|x| \leqq 1, n=1,2, \cdots) .
$$

Prabhu [15] p. 64 has shown that ${ }_{0} p_{0 j}^{(n)}=j f_{n-j}^{(n)} / n(n \geqq j),{ }_{o} p_{0 j}^{(n)}=0$ otherwise, where

$$
{ }_{0} p_{0 j}^{(n)}=\operatorname{Pr}\left\{Q_{n}=j, Q_{m} \neq 0,(m=1, \cdots, n-1) \mid Q_{0}=0\right\} \quad(n=1,2, \cdots) .
$$

It is not difficult to see that

and

$$
\xi(1)=\xi=\sum_{n=1}^{\infty}{ }_{0} p_{01}^{(n)}, \xi^{\prime}(1)=\sum_{n=1}^{\infty} n_{0} p_{01}^{(n)}(n)
$$

$$
\beta_{n}=\sum_{j=1}^{n}{ }_{0} p_{0 j}^{(n)}
$$

thus the $r_{n}$ can be expressed solely in terms of these last exit probabilities.

\section{Discrete time queues}

Meisling [11] has considered a queueing process in which customers can only arrive at time instants which are multiples of $\Delta>0$. At any such instant either no customers arrive, with probability $q>0$, or exactly one customer arrives, with probability $p=1-q>0$. The service times of successive customers are identically and independently distributed lattice random variables with distribution $c_{k}=\operatorname{Pr}$ \{service time $\left.=k \Delta\right\}(k=1,2, \cdots)$. The queue lengths at successive departure epochs form a stochastically monotone Markov chain with one step transition probabilities $p_{i j}=b_{j+1-i}(1 \leqq i \leqq j+1), p_{0 j}=p_{1 j}$, and $p_{i j}=0$ otherwise $(i, j=0,1, \cdots)$, where

$$
b_{i}=\sum_{k=i}^{\infty}\left(\begin{array}{l}
k \\
i
\end{array}\right) p^{i} q^{k-i} c_{k}
$$

When $p q>0$ this Markov chain is irreducible, and if

$$
\sum_{k=1}^{\infty} k c_{k}<1 / p
$$

it is ergodic and Theorem D applies to stationary queues of this nature.

Beusch [2] has considered a queueing system where customers can only arrive at time instants which are multiples of $\Delta>0$, and where the service times are positive multiples of $\Delta$. He studies the process $\left\{X_{n}\right\}$ where $X_{n}$ is the sum of the service times of all customers in the queue at time $n \Delta(n=0,1, \cdots)$ including those customers which arrive at $n$ and the remaining service time of the customer receiving service at this instant. If $Y_{n}$ is the sum of the service times of the customers that arrive at $n$, and if $\operatorname{Pr}\left\{Y_{n}=i\right\}=d_{i}(i=0,1, \cdots ; n=1,2, \cdots)$ and if the $Y_{n}$ 
are all independent, then $\left\{X_{n}\right\}$ is a Markov chain with one step transition probabilities $p_{i j}=d_{j+1-i}(1 \leqq i \leqq j+1), p_{0 j}=p_{1 j}$, and $p_{i j}=0$ otherwise $(i, j=0,1$, $\cdots$. This Markov chain is stochastically monotone and when $d_{0}+d_{1}<1$ it is irreducible and if $\sum_{i=1}^{\infty} i d_{i}<1$ it will be ergodic and Theorem $\mathrm{D}$ can be applied to stationary Markov chains of this type.

It is clear that the transition matrices of the Markov chains just discussed are of a similar form to that of the embedded queue length process in the $M / G / 1$ queue, so that under suitable conditions it seems likely that much of the discussion of Sections 2 and 3 could apply to the Markov chains mentioned above.

\section{Acknowledgements}

I am indebted to the referee for substantial improvements in the presentation of the paper. This work was carried out with the help of a Department of Supply cadetship.

\section{References}

[1] V. E. Beneš, 'On queues with Poisson arrivals', Ann. Math. Stat. 28 (1957), 670-677.

[2] J. V. Beusch, 'A general model of a single-channel queue: Discrete and continuous time cases', Opns. Res. 15 (1967), 1131-1144.

[3] N. Blomqvist, 'The covariance function of the $M / G / 1$ queueing system', Skand. Akt. (1967), $157-174$.

[4] E. W. Cheney, 'Introduction to Approximation Theory' (McGraw-Hill, New York, 1966).

[5] B. D. Craven, 'Serial dependence of a Markov process', J. Aust. Math. Soc. 5 (1965), 299-314.

[6] D. J. Daley, 'The serial correlation coefficient of waiting times in a stationary single server queue', J. Aust. Math. Soc. 8 (1968), 683-699

[7] D. J. Daley, 'Monte Carlo estimation of the mean queue size in a stationary $G I / M / 1$ queue', Opns. Res. 16 (1968), 1002-1005.

[8] D. J. Daley, 'Stochastically monotone Markov chains', Z. Wahr. 10 (1968), 305-317.

[9] J. H. Jenkins, 'Stationary joint distributions arising in the analysis of the imbedded Markov chain of the $M / G / 1$ queue', J. App. Prob. 3 (1966), 512-520.

[10] S. Karlin, A First Course in Stochastic Processes (Academic Press, New York, 1966).

[11] T. Meisling, 'Discrete time queueing theory', Opns. Res. 6. (1958), 96-105.

[12] P. M. Horse, 'Stochastic properties of waiting lines', Opns. Res. 3 (1955), 255-262.

[13] A. G. Pakes, 'On a class of Markov chains', J. Aust. Math. Soc.

[14] E. Parzen, Stochastic Processes (Holden Day, San Francisco, 1962).

[15] N. U. Prabhu, Stochastic Processes (MacMillan, New York, 1965).

[16] J. F. Reynolds, 'On the autocorrelation and spectral functions of queues', J. App. Prob. 5 (1968), 467-475.

[17] T. L. Saaty, Elements of Queueing Theory (McGraw-Hill, New York, 1961).

[18] L. Takács, Introduction to the Theory of Queues (O.U.P., New York, 1962).

Department of Mathematics

Monash University

Melbourne, Australia 